\title{
Alternative fiber optic sensor based on Bragg grating for heart rate monitoring
}

\begin{abstract}
This paper deals on the alternative fiber optic sensor based on fiber Bragg grating (FBG) for use in biomedical applications. FBG measurement probe mounted on the human chest provides respiratory and heart activity monitoring. The big advantage of these sensors are immunity to electromagnetic interference and therefore they are able to be employed in magnetic resonance imaging (MRI) environment. The FBG encapsulation plays an important role in sensor protection against external effects and damages. This article focuses on the various types of FBG encapsulation and implementation on the human body for vital sign monitoring. Presented results of fiber optic sensors show a very interesting alternative to conventional respiratory and heart rate monitors.
\end{abstract}

Keywords: fiber Bragg grating, sensors, respiratory rate, heart rate
Volume 4 Issue 5 - 2018

\author{
Marcel Fajkus,' Jan Nedoma,' Radek \\ Martinek $^{2}$ \\ 'Department of Telecommunications,VSB - Technical university \\ of Ostrava, Czech Republic \\ ${ }^{2}$ Department of Cybernetics and Biomedical Engineering,VSB - \\ Technical university of Ostrava, Czech Republic
}

Correspondence: Marcel Fajkus, Department of Telecommunications, VSB - Technical university of Ostrava, 17. listopadu 15, 70833 Ostrava, Czech Republic, Tel +420 597 326 057, Email marcel.fajkus@vsb.cz

Received: May 31, 2018 | Published: October 09, 2018

\section{Introduction}

A number of research articles which used one or more FBGs have presented results of measurements of respiration rate, heart rate or both simultaneously. ${ }^{1-3}$ Other reports have presented very interesting results based on the design and construction of an FBGbased sensor attached to the patient's chest that enables respiratory rate, heart rate and temperature monitoring. ${ }^{4}$ The experimental results reported in article describe an FBG-based sensor encapsulated into polymer polydimethylsiloxane (PDMS), which is mounted on an elastic contact strap that encircles the patient's chest. ${ }^{5}$ Fiber optic sensors encapsulated into smart textile are henceforth very popular for respiratory rate monitoring capable of operating in MRI environments. ${ }^{6-7}$

\section{Methods}

An optical fiber is a transparent cylindrical fiber made from silica glass. Optical fiber consists of an inner core, an outer cladding and acrylate protection with the outer diameter of $250 \mu \mathrm{m}$. Optical fibers are used to transmit light between the two ends of the fiber and find wide usage in fiber-optic communications. Specially designed fibers are also used for sensory applications. The most common types of fiber sensors are fiber Bragg gratings. Bragg grating is formed by the periodic change of refractive index in the core of the optical fiber. ${ }^{8}$ This structure reflects narrow part of propagation light and transmits other wavelengths (Figure 1). Central part of reflected light is called

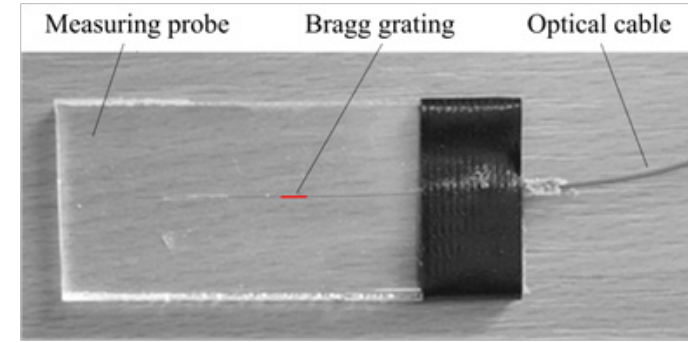

(A)
Bragg wavelength and is given by equation:

$$
\lambda_{B}=2 n_{\text {eff }} \Lambda
$$

where $n_{\text {eff }}$ is effective refractive index of periodic structure in core of optical fiber and $\Lambda$ is period between these periodic changes. For biomedical applications, Bragg gratings are encapsulated into polymer polydimethylsiloxane (Figure 2A) and placed on the chest and fixed by a contact elastic strap. Another type of Bragg measurement probe consists of fiber Bragg grating located between two layers in process of print with 3D printer (Figure 2B). The source used for measurement is the wide spectral LED (Light Emitting Diode). Next, there is a circulator that directs light to Bragg grating, and reflected Bragg wavelength directs into OSA (Optical Spectrum Analyzer) where shifts of Bragg wavelengths are analyzed in the spectral domain.

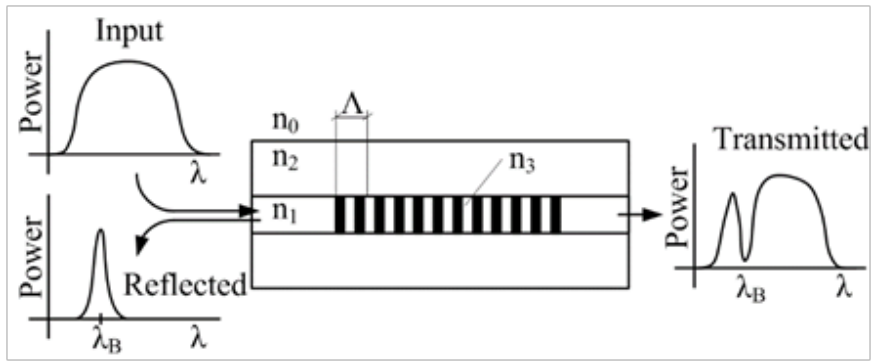

Figure I Operating principle of fiber Bragg grating.

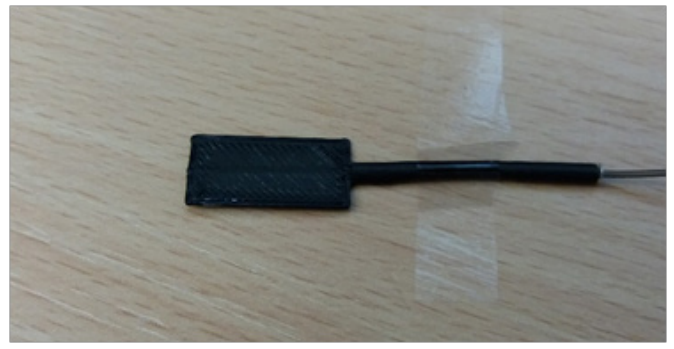

(B)

Figure 2 Measurement probe with Bragg grating encapsulated into PDMS (A); FBG measurement probe fabricated in 3D printer. 


\section{Results}

The measurement probes were placed on the chest under the elastic strap. The strap was relatively tightened to increase the sensitivity of the probes. Figure 3A shows the measured data where the breathing activity is noticeable. Each separate peak is the maximal inflation

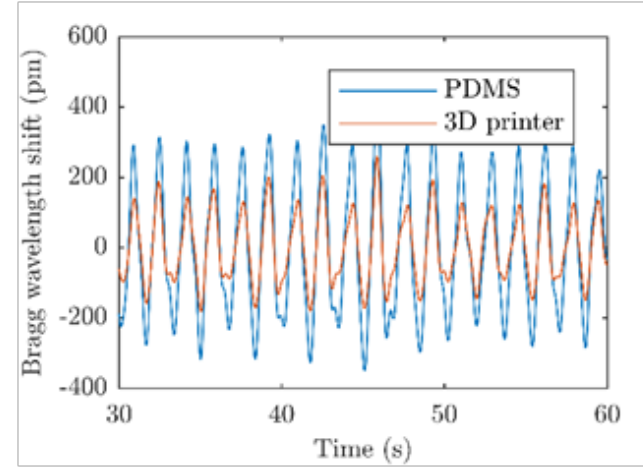

(A) of the patient and the subsequent descent is the gradual exhalation. For further processing, a band-pass filter of the 10th order with a range from 5 to $20 \mathrm{~Hz}$ was used for heart activity derivation. Figure 3B shows the results of the filtration. In the first second, there is a synchronization pulse, and there are noticeable periodic repetitions that represent the pulse activity.

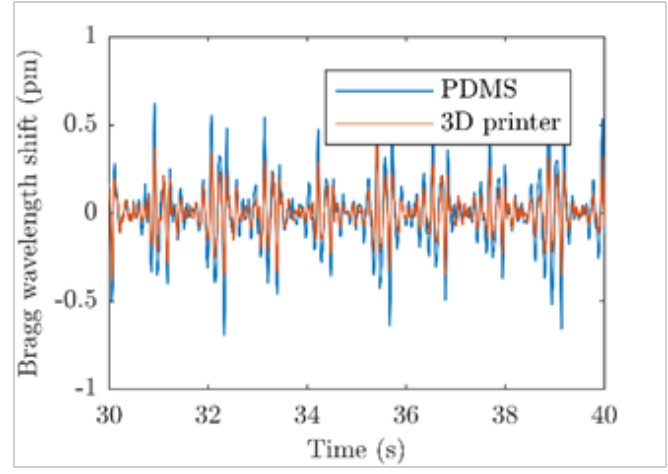

(B)

Figure 3 Respiratory activity from measurement probes without filtration (A); Heart activity signal from measurement probes after filtration by the band pass filter.

Heart rate from both measurement probes based on fiber Bragg grating and reference ECG is shown in Figure 4A \& Figure 4B. To compare the differences between the reference signals from ECG and those acquired from both measurement probes, the Bland-Altman plot was utilized..$^{9}$ The differences between the sensor and the reference traces, $x_{1}-x_{2}$, are plotted against the average, $\left(x_{1}+x_{2}\right) / 2$, where $\mathrm{x}_{1}$ is reference heart rate from ECG and $\mathrm{x}_{2}$ is heart rated determine from FBG measurement probes. The reproducibility is considered to be good if $95 \%$ of the results lie within the $\pm 1.96 \mathrm{SD}$ (Standard Deviation) range.

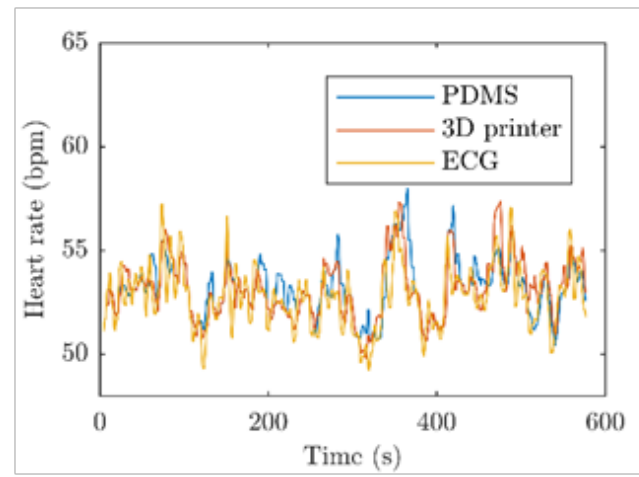

(A)

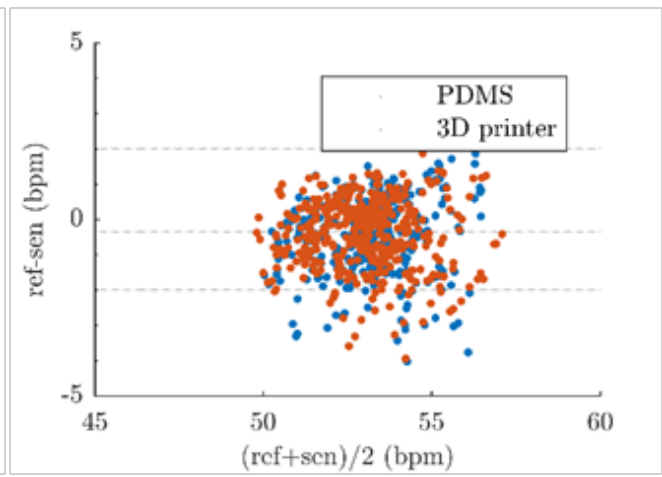

(B)

Figure 4 Heart rate calculated from both FBG measurement probes and reference ECG (a); Bland-Altman statistic for heart rate measurement with FBG measurement probes.

\section{Conclusion}

This article describes a alternative non-invasive fiber-optic sensor based on the fiber Bragg gratings for the monitoring of a patient's heart rate and respiratory rate. The Bland-Altman statistical analysis demonstrates no basic systematic errors. For the entire data set 96.49 $\%$ (for PDMS probe) and $95.4 \%$ (for printed probe) of the values lie within the $\pm 1.96 \mathrm{SD}$ range for the heart rate determination. The results of heart rate measurements are characterized by the maximum relative error of $4.67 \%$. These results can help to predict hyperventilation and panic attacks of patients during MRI examination.

\section{Acknowledgements}

This article was supported by the Ministry of Education of the Czech Republic (Project No. SP2018/184).

\section{Conflict of interests}

Authors declare that there is no conflict of interest.

\section{References}

1. Chethana K, Guru Prasad AS, Omkar SN, et al. Fiber bragg grating sensor based device for simultaneous measurement of respiratory and cardiac activities. J Biophotonics. 2017;10(2):278-285.

2. Dziuda L, Skibniewski FW, Krej M, et al. Monitoring respiration and cardiac activity using fiber Bragg grating-based sensor. IEEE Trans Biomed Eng. 2012;59(7).

3. Fajkus M, Nedoma J, Siska P, et al. FBG sensor of breathing encapsulated into polydimethylsiloxane. SPIE - The International Society for Optical Engineering. 2016. 
4. Fajkus M, Nedoma J, Martinek R et al. A non-invasive multichannel hybrid fiber-optic sensor system for vital sign monitoring. Sensors (Switzerland). 2017;17(1).

5. Nedoma J, Fajkus M, Martinek R, et al. Non-invasive fiber-optic biomedical sensor for basic vital sign monitoring. Adv Electr Electron Eng. $2017 ; 15(2)$.

6. Witt J, François Narbonneau, Marcus Schukar, et al. Medical textiles with embedded fiber optic sensors for monitoring of respiratory movement. IEEE Sens J. 2012;12(1).
7. Presti DL, Carlo Massaroni, Domenico Formica, et al. Smart Textile Based on 12 Fiber Bragg Gratings Array for Vital Signs Monitoring. IEEE Sens J. 2017;17(18):6037-6043.

8. Othonos A. Fiber bragg gratings. Rev Sci Instrum. 1997;68(12):43094341.

9. Bland JM, Altman DG. Measuring agreement in method comparison studies. Stat Methods Med Res. 1999;8(2):135-160. 